\title{
Ultrasonic Reflection Tomography Vs. Canonical Body Approximation: Experimental Assessment of an Infinite Elastic Cylindrical Tube
}

\author{
Philippe Lasaygues ${ }^{1}$ and Loïc Le Marrec ${ }^{2}$ \\ ${ }^{1}$ Laboratory of Mechanics and Acoustics (LMA) \\ UPR CNRS 7051 \\ Marseille, France \\ lasaygues@lma.cnrs-mrs.fr \\ ${ }^{2}$ Institute of Mathematical Research of Rennes (IRMAR) \\ UMR CNRS 6625 \\ Rennes, France
}

\begin{abstract}
Comparisons were made between the results obtained using two quantitative ultrasound imaging methods on the solid cross section of a cylindrical tube that is infinite in the axial direction. The first method tested was the classical reflection tomography method based on the first-order Born approximation, which can only be used under conditions to obtain limited reconstruction of the external boundaries of the high contrast scatterer. The results were compared with those obtained using another inversion scheme based on the Intercepting Canonical Body Approximation (ICBA) in a large frequency range, which gives accurate complete geometrical information about the tube (thickness measurements). The numerical and experimental results obtained show the feasibility of the latter approach.
\end{abstract}

Key words: Born approximation; canonical approximation; elastic tube; quantitative imaging.

\section{INTRODUCTION}

The development of ultrasonic characterization and imaging methods for use on elastic cylindrical tubes is of great importance in several medical and technical fields, where these methods are required for bone imaging and nondestructive testing of steel tubes, for example. In the case of elastic tubes showing high impedance contrast with the surrounding medium (soft tissues in medical applications, water or coupling gel in nondestructive testing), ultrasonic propagation is greatly perturbed, which gives rise to imaging problems.

Many authors have dealt with ultrasonic characterization and imaging of elastic tubes. The aim of most previous studies has been to assess the thickness of the tube (as part of its geometrical conformation) and to calculate the speed of sound (an acoustical parameter) of the compressional wave crossing the structure. To study the shape of elastic tubes, measurements can be made in a plane parallel to the generator axis (in the $z$-direction), using an 'axial or transverse measurement' procedure, or in a perpendicular plane, using a 'radial or cross-sectional measurement and imaging' procedure. Our group is particularly interested in the cross-sectional imaging method, which we have approached as a problem where ultra- 
sonic waves are scattered by a solid cylindrical cavity. The ultimate aim is to find a means of solving inverse scattering problems in the case of tubes. One of the prerequisites to to achieve this aim is to solve the corresponding forward problems. Several methods have been applied to solving forward problems, including integral equation methods, hybrid finite-elements methods and the geometrical theory of diffraction. The latter method provides asymptotic approximations for diffracted fields, which are valid at high frequencies and at large distances from the diffracting body. Several imaging strategies are possible in this context but to obtain real time imaging, simple, efficient and accurate numerical methods of modeling the forward and inverse problems are required.

Our Ultrasonic Reflection Tomography (URT) method, based on the inverse Born approximation, is one of these strategies. ${ }^{1}$ This method has proved to be accurate and gives promising results in terms of the shape of the tubes tested (qualitative tomography). In the case of materials showing high acoustical impedance contrast with the surrounding medium, however, it is not possible to reconstruct the inner cavity or to determine the acoustical parameters (such as the velocity and/or the attenuation of the sound waves) without correcting for wave propagation through the water/shell interface. The main reason for this problem is that the Born approximation solving the forward problem is valid for objects showing a weak impedance contrast with the surrounding medium (high frequency approximation). Modeling an elastic tube with a model where the acoustic properties are too similar to those of the surrounding medium results in tomograms containing geometrical aberrations as far as the dimensions of the shell are concerned.

In this paper, a new model based on a canonical approximation is presented for solving the forward problem. The modeling procedure used here is based on the Intercepting Canonical Body Approximation (ICBA) method developed by A. Wirgin and his group..$^{2-4}$ In contrast to straight ray theory, no limited configuration of the scattered field is required. The ICBA method consists in replacing the real scatterer at each observation point by an object having simple geometrical characteristics and known acoustical properties, and the solution of the forward problem has to be canonical (i.e., it has to be previously determined analytically). For example, in the case of a noncircular elastic tube, a circular tube having the same inner and outer radii as the local radii of the real object in the measurement direction would provide a suitable model.

Here we deal with the case of an elastic cylindrical tube, which is infinite in the $z$-direction and is immersed in a homogeneous fluid, namely water. Note that the real object is not necessary centered or circular, as required in the case of the model used for ICBA. Its cavity is taken to be weakly heterogeneous in comparison with the wavelength. In fact, we consider weak the local fluctuations in the acoustic characteristics of the cross-section of the scatterer.

This is an iterative tomographic approach requiring preliminary a priori information. We distinguish between high-level a priori knowledge about parameters such as the density, velocity of the waves propagating in the shell, and thickness of the 'medullar' canal, and low-level a priori information about parameters such as the outer radius or acoustic parameters of the surrounding medium. We will therefore focus on determining the thickness of the tube by studying the resolution of the images. Other physical parameters such as the densities and velocities are set in this work at their exact values. A sinogram (diffracted angle vs. time) based on experimental measurements and numerical simulations is drawn up. The cross-sectional image of the tube is first determined using URT and a filtered back-projections algorithm. ${ }^{5}$ The determined external boundary of the scatterer is then used as the low-level a priori information. The second approach (that based on ICBA) was developed in order to optimize the inverse problem. The accuracy of the boundaries and, hence, the thickness is thus improved. The particular case of a tube having a similar thickness to the wavelength of the wave in the water will be studied. 
The classical ultrasonic tomography method is described in section 3. The ICBA approach to the forward and inverse problem is presented in section 3 and results obtained on numerical and experimental scatterers are given. The results presented in section 4 are of considerable importance in the field of ultrasonic imaging.

\section{CONFIGURATION AND STATEMENT OF PROBLEM}

\section{Physical considerations}

The elastic and acoustic properties of the material in the cross-section of the tube are taken to be isotropic. This elastic scatterer mediates the propagation of complex elastic volume waves, namely compressional P-waves and shear vertical SV-waves (shear horizontal $\mathrm{SH}$-waves are not taken into account here). Since the acoustic impedance of the tube contrasts strongly with that of the surrounding water (the scatterer is immersed in a water tank), the ultrasonic propagation is greatly perturbed by refraction, attenuation and scattering of the waves. ${ }^{6}$

The frequency bandwidth used here in the cross-section imaging was $0.25-1 \mathrm{MHz}$. The wavelengths occurring in the tube - typically $2-16 \mathrm{~mm}$ at compression waves velocities ranging between 2,000 and $4,000 \mathrm{~m} / \mathrm{s}$ - were therefore much greater than the macroscopic porosity and the microstructural scale of the shell $(\approx 1 \mathrm{~mm})$. The tube was therefore taken to be weakly heterogeneous (small perturbations of the propagation) and to consist of a homogenized equivalent medium. It was assumed that the ultrasonic wave in the shell was not disturbed by the microstructure. The latter assumption is necessary to be able to introduce a linearized propagating theory and to adopt an asymptotic approximation. On the other hand, the wavelength $(1.5,6 \mathrm{~mm})$ in water $\left(c_{0} \approx 1,500 \mathrm{~m} / \mathrm{s}\right)$ is smaller than the diameter $(\approx 10 \pm 2$ $\mathrm{mm}$ ) of the object. The $k a$ product, where $k=\omega / c$ is the wave number and $a$ is the mean-radius of the tube, ranges between 6and 25 and the configuration is therefore nonresonant.

\section{Geometrical considerations}

In this section, it is proposed to study the homogeneous noncircular solid tube, the physical properties of which are known. The canonical 2-D geometry considered here is shown in figure 1 . The surrounding medium consists of a linear, homogeneous, uncompressible fluid (a water-like fluid, for example). The noncircular elastic cylindrical cavity has generators placed parallel to the $z$-axis and is immersed in the surrounding medium. We define in the $x y$-plane: $\rho_{o}$ the density and $c_{o}$ the velocity of the propagating wave in the surrounding medium and the hollow area, $\rho_{1}$ the density of the cavity and $c_{l p}$ and $c_{l t}$ the velocity of the propagating longitudinal and transversal waves. We take $r_{l}\left(\theta_{m}\right)$ and $r_{2}\left(\theta_{m}\right)$, to denote the radius of the external and internal boundaries, respectively, of the cavity at the observation angle $\theta_{m}$.

It will be assumed that high-level a priori knowledge is used for $\rho_{l}$ and $c_{l}$. The external radius $r_{l}$, and internal radius $r_{2}$ are unknown and the aim of this study is to determine these radii. However, we assume that these radii are included in a limited set of values (corresponding to the low level a priori information).

\section{ULTRASONIC SCATTERING PROBLEM}

Here, we deal with the forward scattering problem, i.e., predicting the scattered field when the scattering medium and incident field are assumed to be known, as well as with the inverse 


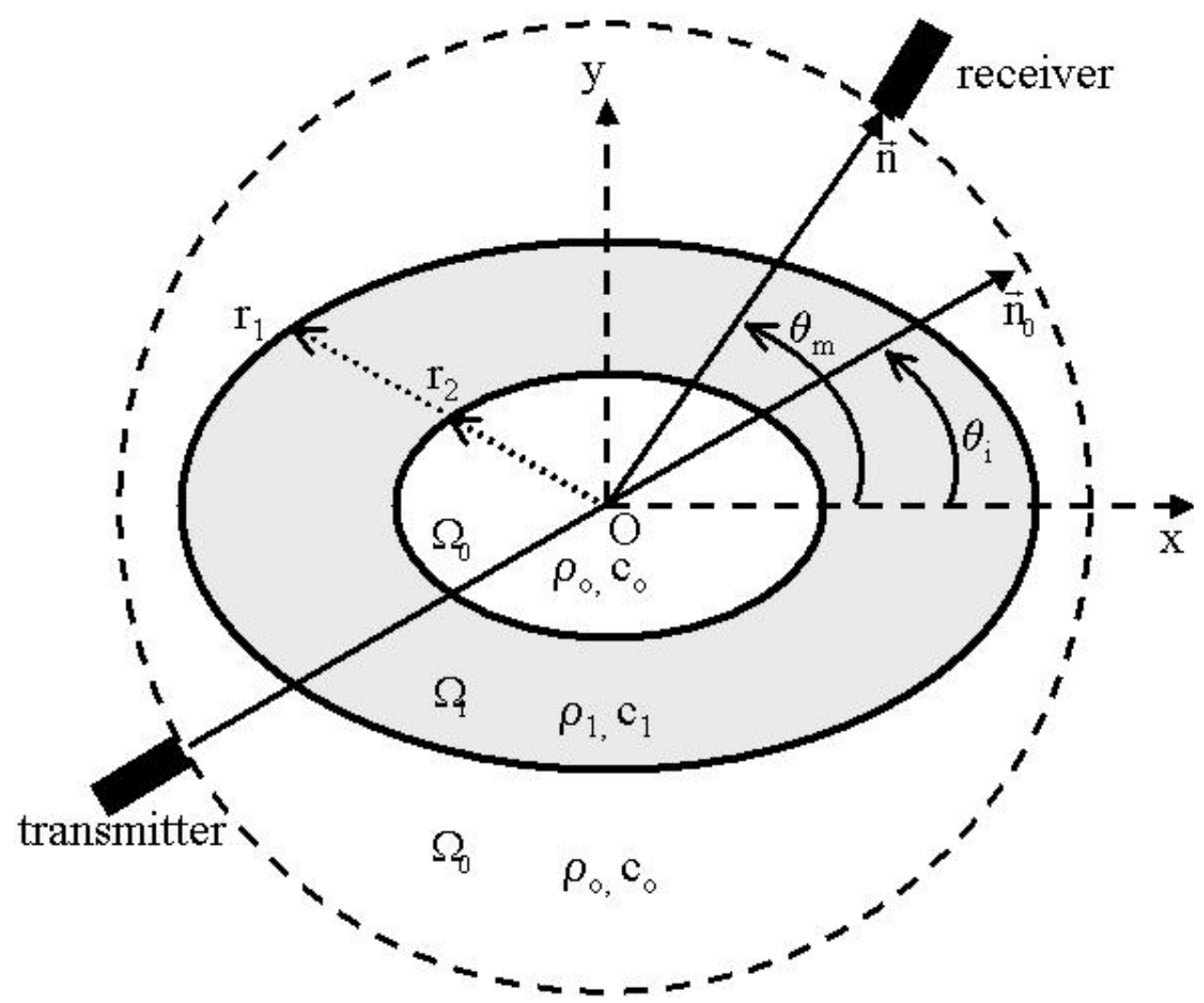

FIG. 1 Noncircular cylindrical elastic cavity: geometrical and acoustical configuration.

scattering problem, i.e., retrieving the boundaries of the medium from the measured (or simulated) incident and scattered fields, all the material properties being known. First, we look at the forward problem in order to obtain the state equation, which is essential to solving the inverse problem.

Since inverse problems are nonlinear and ill posed, one of the consequences is that there is no single solution. In most ultrasonic scattering problems, approximate models (such as the first-order Born approximation or canonical approximation) ${ }^{3}$ are used and it is generally necessary to find a means of eliminating solutions, which do not correspond to the real parameters (Delamare et al. ${ }^{7}$ have proposed a technique based on Born approximation). To detect the boundaries of a high-contrast cylindrical cavity, we establish that:

1. The scattering problem can be linearized using the first-order Born approximation method. If the Green function of the unperturbed problem (the surrounding medium $\Omega_{0}$ ) is known, we can solve the direct problem with the Lippmann-Schwinger integral equation and the far field solution of the equation can be calculated. The inverse problem can then be solved using a tomographic algorithm to obtain a perturbation map with respect to the reference problem.

2. A rational method of improving the resolution consists in combining canonical approximation with the analysis of a functional (cost-function) measuring the gap between the scattered and predicted fields. The functional chosen for this purpose is generally the least-mean square estimate obtained as follows:

$$
F\left(\tau_{m} ; \vec{x}_{m} ; \omega, \Phi\right)=\left|P^{P}\left(\vec{x}_{m} ; \omega, \Phi\right)-P^{e}\left(\tau_{m} ; \vec{x}_{m} ; \omega, \Phi\right)\right|^{2}
$$


where $P^{P}\left(\vec{x}_{m} ; \omega, \Phi\right)$ is the predicted and $P^{e}\left(\tau_{m} ; \vec{x}_{m} ; \omega, \Phi\right)$ the estimated scattered field. These fields are function of the pulsation $\omega$, the observation point $\vec{x}_{m}=\left(r_{\mathrm{m}}, \theta_{\mathrm{m}}\right)$ and incident angle $\theta_{i}$, this latter parameter being expressed through the diffracted angle $\phi=\theta_{\mathrm{I}}-\theta_{m}$. The estimated field depends on the unknowns (radii) it is proposed to reconstruct. We denote these unknowns using general term, the vector $\tau_{\mathrm{m}}$.

As a canonical approximation of the interactions between the wave and the body, the Intercepting Canonical Body Approximation (ICBA) has proved to be an efficient forward solver both in and beyond the resonant domain in the case of full and hollow elastic cylinders. We will see below how an ICBA extrapolation of the nonresonance diffraction can be used to image the shape of the cavity.

\section{Born modeling and reflection tomography}

The goal of ultrasonic tomography is to reconstruct the spatial distribution of some of the geometrical parameters of an object from scattered ultrasonic measurements. These measurements are carried out at variably-dense sets of transmitter and receiver positions and interrogating wave frequencies.

The inverse scattering problem is solved using a Born approximation, which gives a particularly simple and attractive linear relation between the object function and the scattered field, particularly in the far-field (2D or 3D Fourier transform). This makes it possible, in principle, to reconstruct the object function practically in real time when a sufficiently large set of scattering data is available.

\section{Asymptotic considerations}

The basic principles of URT have by now been clearly established in the case of weaklyvarying media such as low-contrast structures, i.e., almost homogeneous media. ${ }^{8}$ The reference medium chosen can therefore be a constant medium (forming a constant background), yielding an Inverse Born Approximation (IBA). In the case of our configuration, we take $\Omega_{0}$ to denote the known background expressed in terms of the density $\rho_{0}$ and the velocity $c_{0}$, and $\Omega_{1}$ to denote the unknown perturbation (scatterer) expressed in terms of the homogeneous density $\rho_{1}$ and the velocity $c_{1}$.

Under weak scattering conditions $\left(\rho_{1} c_{1} \approx \rho_{0} c_{0}\right)$, the following temporal equation based on the Pekeris equation describes the acoustic propagation processes occurring in the medium (including the boundary and Sommerfeld conditions):

$\left[-\frac{1}{c_{0}^{2}} \frac{\partial^{2} \cdot}{\partial t^{2}}+\Delta \cdot\right] p\left(\vec{x}_{m}, t\right)=-\frac{\alpha\left(\vec{x}_{m}\right)}{c_{0}^{2}} \frac{\partial^{2} p\left(\vec{x}_{m}, t\right)}{\partial t^{2}}+\operatorname{gra} d p\left(\vec{x}_{m}, t\right) \cdot \operatorname{gr} \vec{a} d \xi\left(\vec{x}_{m}\right)-\frac{1}{2} \operatorname{gr} \vec{a} d p\left(\vec{x}_{m}, t\right) \cdot \operatorname{gr} \vec{a} d \alpha\left(\vec{x}_{m}\right)$

where $p\left(\vec{x}_{m}, t\right)$ is the acoustic pressure.

$\alpha_{x}=\left(c_{1}^{2}(\vec{x})-c_{0}^{2}\right) / c_{1}^{2}(\vec{x})$ is the quadratic fluctuation of velocity and $\xi(\vec{x})=$ $\log \left(Z_{1}(\vec{x}) / Z_{0}\right)$ is the logarithmic fluctuation of acoustic impedance, $Z_{1}(\vec{x})=\rho_{1}(\vec{x}) c_{1}(\vec{x})$.

If the Green function of the nonperturbed problem is given, we can find the total pressure field in $\Omega_{\mathrm{o}}$ using the Lippmann-Schwinger integral equation:

$$
p\left(\vec{x}_{m}, t\right)=p^{i}\left(\vec{x}_{m}, t\right)+p^{s}\left(\vec{x}_{m}, t\right)
$$

where $p^{s}\left(x_{m}, t\right)$ denotes the scattered field in $\Omega_{\mathrm{o}}$ given by: 
$p^{s}\left(\vec{x}_{m}, t\right)=-\int_{R^{3}} \frac{1}{4 \pi\left|\vec{x}_{m}-\vec{x}^{\prime}\right|}\left\{\frac{\alpha}{c_{0}^{2}} \frac{\partial^{2} p}{\partial t^{2}}+\text { gra } d p \cdot g r \vec{a} d \xi-\frac{1}{2} \operatorname{gra} d p \cdot g r \vec{a} d \alpha\right\}_{\left(\vec{x}_{m}, t-\frac{\left|\vec{x}_{m}-\vec{x}^{\prime}\right|}{c_{0}}\right)} d^{3} \vec{x}^{\prime}$

One can calculate the far field solution of this equation in the direction $\vec{n}=\vec{x}_{m} / \mid \vec{x}_{m}$ in the case of an ideally pulsed plane-wave insonification, with the incidence $\vec{n}_{0}=\vec{x}_{0} /\left|\vec{x}_{0}\right|$ :

$$
p^{i}\left(\vec{x}_{m}, t\right)=\delta\left(t-\frac{\vec{n}_{0} \cdot \vec{x}_{m}}{c_{0}}\right)
$$

Under backscattering conditions $\left(\vec{n}=-\vec{n}_{0}\right)$ and within the first-order Born approximation (single-scattering), the asymptotic solution of Eq.(3) is:

$$
p^{s}\left(\vec{x}_{m}, t\right)=\frac{1}{4 \pi\left|\vec{x}_{m}\right|} h_{d(1)}^{\infty}\left(\vec{x}_{m}, t-\frac{\left|\vec{x}_{m}\right|}{c_{0}}\right)
$$

where $h_{d(1)}^{\infty}$ is the first-order far-field response of the medium:

$$
h_{d(1)}^{\infty}\left(\vec{x}_{m}, t\right)=\frac{c_{0}}{4} \frac{\partial}{\partial x_{3}^{2}}\left\{\int_{R^{2}} \xi\left(\vec{x}_{3}^{\prime}\right) d^{2} \vec{x}\right\}_{x_{3}=\frac{c_{0} t}{2}}
$$

where $x_{3}$ is the $\vec{n}_{0}$ axis. In this case, the parameter $\alpha$ is eliminated.

In the space-frequency domain, the backscattering transfer function is given by:

$$
H_{d(1)}^{\infty}\left(\vec{n}_{0}, \omega\right)=-2 k^{2} \hat{\xi}(\vec{K})
$$

where

$$
\hat{\xi}(\vec{K})=\int_{R^{3}} \xi(\vec{x}) e^{-i \vec{K} \cdot \vec{x}} d^{3} \vec{x}
$$

is the Fourier transform of the logarithmic fluctuation of the acoustic impedance and $\vec{K}=-2 k \vec{n}_{0}$ is the wave vector.

If one takes only the amplitude of the echoes in account, one obtains $a_{d(1)}^{\infty}\left(\vec{n}_{0}, \omega\right)=\hat{r}(\vec{K})$, where $\hat{r}(\vec{K})$ is the spatial Fourier transform of the reflectivity of the medium $r(\vec{x})$.

The purpose of URT is to obtain reflectivity images from backscattering measurements. Rotating the emitter-receiver transducer around the 'object' and emitting broadband pulses at each position leads to the same situation as in X-ray tomography; one obtains slice-byslice spectral coverage of the object spectrum $\hat{r}(\vec{K})$. Any tomographic reconstruction algorithm can therefore be used to perform the reconstruction. We chose an algorithm of summation of the backprojections of filtered projections. ${ }^{9}$ 


\section{Tomography of a high-impedance contrast scatterer}

In the ultrasonic characterization of high contrast bodies, it is not possible to use the IBA with a 'constant' background. For example, hard biological tissues (such as bones) are quite heterogeneous and their acoustical characteristics are very different from those of the surrounding soft tissues (or water in vitro). The problem arising with the tube-thickness imaging is how to identify a water-like cavity within an elastic cylinder. In this case, an IBA with a 'variable background' can be used. The background can be defined in terms of the following two parts: on the one hand, a solid part without hollow/water, and on the other hand, the perturbation, i.e., the object to be reconstructed, namely, the cavity. Based on the acoustical assumptions adopted, the shell is weakly heterogeneous (in comparison with the wavelength) and therefore gives off weak echoes. IBA can therefore be used to obtain a good approximation for the inverse scattering problem. The algorithm of tomographic reconstruction by summation of the filtered backprojections can be used with some signal processing refinements. ${ }^{10}$ Due to the high contrast impedance, artifacts are generated in the image by the scattering process (the Born approximation is a single-scattering theory). However, the main result will be preserved: the basis underlying the 'perturbation' will be detected, i.e., a qualitative image of the cavity will be obtained.

\section{Wide-band imaging using canonical approximation}

\section{Analytical considerations}

In this section, we deal with the ultrasonic imaging of 2-D elastic media based on multifrequency, multiview synthetically- and/or experimentally-generated data. The goal is to determine a geometrical parameter of the non-circular elastic tube cross-section from measurements of the scattered pressure field resulting from interactions between the scatterer and the known incident wave. We focus on the high-frequency domain, where the object is larger than the wavelength of the ultrasonic wave $(\mathrm{ka}>1)$.

In our configuration, at observation point $\vec{x}_{m}$ in $\Omega_{o}$, we write the total pressure field:

$$
P\left(\vec{x}_{m}, \omega, \Phi\right)=P^{i}\left(\vec{x}_{m}, \omega, \Phi\right)+P^{s}\left(\vec{x}_{m}, \omega, \Phi\right)
$$

where $P^{s}\left(\vec{x}_{m} ; \omega, \Phi\right)$ is the Fourier transform of $p^{S}\left(\vec{x}_{m}, t\right)$ and denotes the scattered field in $\Omega_{o}$ and $P^{i}\left(\vec{x}_{m} ; \omega, \Phi\right)$ is the Fourier transform of $p^{i}\left(\vec{x}_{m}, t\right)$ and denotes the incident field expressed by:

$$
P^{i}\left(\vec{x}_{m}, \omega, \Phi\right)=P_{o} e^{i \vec{k} \cdot x_{m}}
$$

where $\omega$ is the pulsation and $\vec{K}$ is the wave vector in the surrounding medium $\Omega_{o}$.

The acoustic fields are governed by the Helmholtz equation and the scattered field satisfies the usual conditions (Sommerfeld radiation conditions, continuity of pressure and normal velocities at the interface).

The inverse problem is approached using the 'Intercepting Canonical Body Approximation' (ICBA) method developed by A. Wirgin et al to solve the inverse scattering problem in the resonance domain. Knowing the position of the receiver in relation to the emitted ultrasound source, it is assumed that the scattering process at observation point $\vec{x}_{m}$ is identical to that which would have been produced by an acoustically identical circular cylinder with an 'apparent' radius equal to the radius of the object in the measurement direction subjected to 
the same conditions of insonification. The main advantage here is that this procedure can be used even in cases where there is a high impedance contrast.

The estimated field $P^{\mathrm{e}}$ is the field scattered from a centered circular cylinder having the same density $\rho_{1}$ and the same velocity $c_{1}$ as the object and radius $r_{1}^{e}$ equal to the local radius of the object in the measurement direction $\theta_{\mathrm{m}}$. Using the partial wave expansion method, we obtain the following expression for $\vec{x}_{m}=\left(r_{\mathrm{m}}, \theta_{\mathrm{m}}\right) \in \Omega$ :

$$
\begin{aligned}
& P^{e}\left(\vec{x}_{m}, f\right)=A(f) \quad \sum_{n=0}^{N} B_{n}\left(\theta_{m}, f\right) i^{n} \quad \varepsilon_{n} \quad H_{n}^{(1)}\left(k_{0} r_{m}\right) \cos \left(n\left(\theta_{i}-\theta_{m}\right)\right), \\
& N \text { s.t. } \sum_{n=N-10}^{N}\left\|b_{n}\right\|<10^{-4}
\end{aligned}
$$

where $k_{0}=2 \pi f / c_{0}, H_{n}^{(1)}$ is the first-kind Hankel function of the order $n, \varepsilon_{n}$ is the Neumann factor $\left(\varepsilon_{n}=2\right.$ for $\left.n>1\right)$, and the scattering coefficient $B_{n}$ is exactly evaluated using the Rayleigh-Fourier method. ${ }^{11}$

To estimate the unknown $r_{m}=r_{1}^{e}\left(\theta_{m}\right)$, we seek to minimize the cost function,

$$
F\left(\tau_{m} ; \quad \vec{x}_{m}, f\right)=\left|P^{P}\left(\vec{x}_{m}, f\right)-P^{e}\left(\tau_{m} ; \vec{x}_{m}, f\right)\right|^{2}
$$

Since a broadband incident pulse is used, a set of backscattering measurements at $L$ consecutive frequencies (corresponding to $99 \%$ of the pulse spectrum) is available for a given $\theta_{m}$. If these multifrequency data are treated independently, the number of admissible solutions will increase. In the same way, searching for the minima of each of the cost function corresponding to the various frequencies and then applying a postprocessing algorithm to determine the unique solution requires as many minimizations as frequencies. This is also the case with methods based on an iterative frequency algorithm. Whereas the method presented here is based on the minimization of a single cost function, the 'mean cost function' is defined by

$$
\breve{F}\left(\tau_{m} ; \quad \vec{x}_{m}\right)=\frac{1}{L} \sum_{l=1}^{L} F\left(\tau_{m} ; \quad \vec{x}_{m}, f_{l}\right)
$$

\section{Numerical simulation}

To evaluate the validity of the method, we first performed imaging on the basis of synthesized data. The measurements are obtained by solving the acoustic wave propagation problem in 2-D numerically. The method used for this purpose was based on mixed finite elements for the space discretization and on a centered 2 nd order finite difference scheme for the time discretization. ${ }^{12}$

The object is a noncircular cylinder, the cross-section of which is shown in figure 5 . The acoustical properties used in the simulations are $\rho_{0}=1,000 \mathrm{~kg} / \mathrm{m}^{3}, c_{0}=1,500 \mathrm{~m} / \mathrm{s}, \rho_{1}=1,800$ $\mathrm{kg} / \mathrm{m}^{3}$ and $c_{1}=4,000 \mathrm{~m} / \mathrm{s}$. Note that in this simulation, all the materials are fluid-like and, in particular, the object is not elastic.

$P^{P}\left(\vec{x}_{m}, f\right)$ is measured at 16 points equally distributed on a centered measurement ring with radius $r_{m}=12 \mathrm{~mm}$ (near field). The waveform of the incident pulse is a normalized second derivative of a Gaussian function. Two probing pulses are simulated: the 'low frequency 


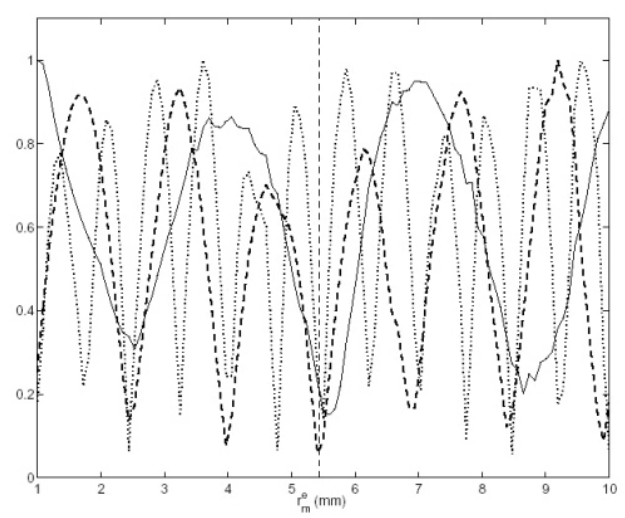

(a)

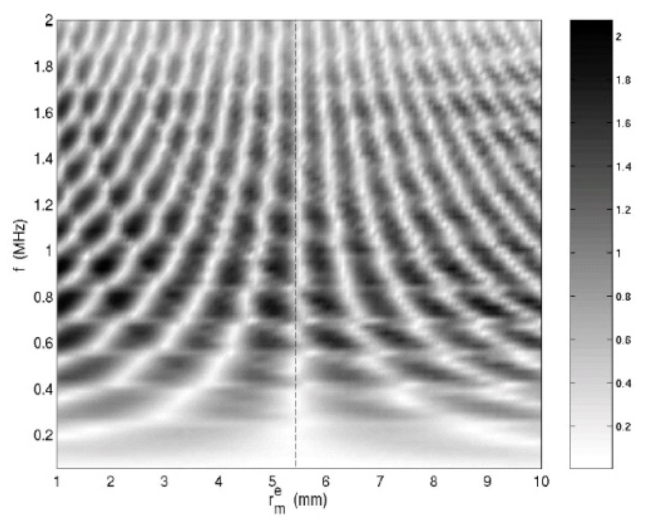

(b)

FIG. 2 Reconstruction of $\mathrm{r}_{l}(\pi / 4)$. (a) amplitude of cost function at three frequencies, $f_{l}($ solid $), f_{2}(\mathrm{dash})$ and $f_{3}(\mathrm{dot})$. (b) single frequency cost function as a function of frequency and estimation parameter (HFI).

insonification' (LFI) pulse with a central frequency $f_{c}=250 \mathrm{kHz}$ and the 'high frequency insonification' (HFI) with $f_{c}=1 \mathrm{MHz}$. Since the mean radius of the object is $6 \mathrm{~mm}$, the first insonification corresponds to an excitation in the resonance region $(k a \approx 6)$ while the second insonification is beyond the resonance region $(k a \approx 25)$. Since ICBA is a monochromatic forward solver, the time-domain measurements are converted into frequency domain data using a Fast Fourier Transform algorithm.

\section{Frequency behavior}

To analyze the role of the frequency in the inversion algorithm, we first compute the single frequency cost function (Eq.(12)) at different frequencies. The parameter of interest is the local radius $r_{1}(\pi / 4)=5.4 \mathrm{~mm}$ and the data are the scattered field measured at $\theta_{\mathrm{m}}=\pi / 4$ (i.e., backprojection, $\left.\theta_{i}=\pi+\theta_{m}\right)$.

Figure $2 \mathrm{a}$ gives the cost function for three different frequencies, $f_{1}=250 \mathrm{kHz}, f_{2}=500 \mathrm{kHz}$ and $f_{3}=1 \mathrm{MHz}$. Note that each of the cost functions has a minimum (not always global) value, which is near the exact solution. However, other estimates (e.g., $r_{1}^{e}=2.5 \mathrm{~mm}$ ) also correspond to the minima of all the cost functions. Careful postprocessing is therefore required when using this algorithm with a small number of frequencies.

Upon plotting the local cost function vs. all the frequencies of HFI (Fig. 2b), it was observed that at a given frequency, a periodic distribution of the minima occurs and the period corresponds to the half wavelength. The attraction domain of each solution can be said to be one half wavelength wide and to be centered on each admissible solution. Moreover, a line of local minima can be observed at which the estimated parameter is approximately equal to the solution and varies weakly with the frequency. This suggests that the reconstruction will never be perfect but that an approximate solution can be obtained with a resolution smaller than the wavelength.

Figure 3 gives the mean cost function Eq.(13) corresponding to the reconstruction of the local radius with HFI. The sum in Eq.(13) is based on three frequency domains $D_{j=1,2,3}$ centered on the central frequency and cover $33 \%, 66 \%$ and $99 \%$, respectively, of the available frequency range. The variations in the cost function decrease and the minimum corresponding to the optimum solution becomes more obvious (i.e., easier to estimate) as the frequency range increases. Averaging over the frequency has a constructive effect on the minimum, which is frequency invariant, and a destructive effect on the others. Moreover, the fact that the attraction domain of the global minimum conserves the same width in all frequency do- 


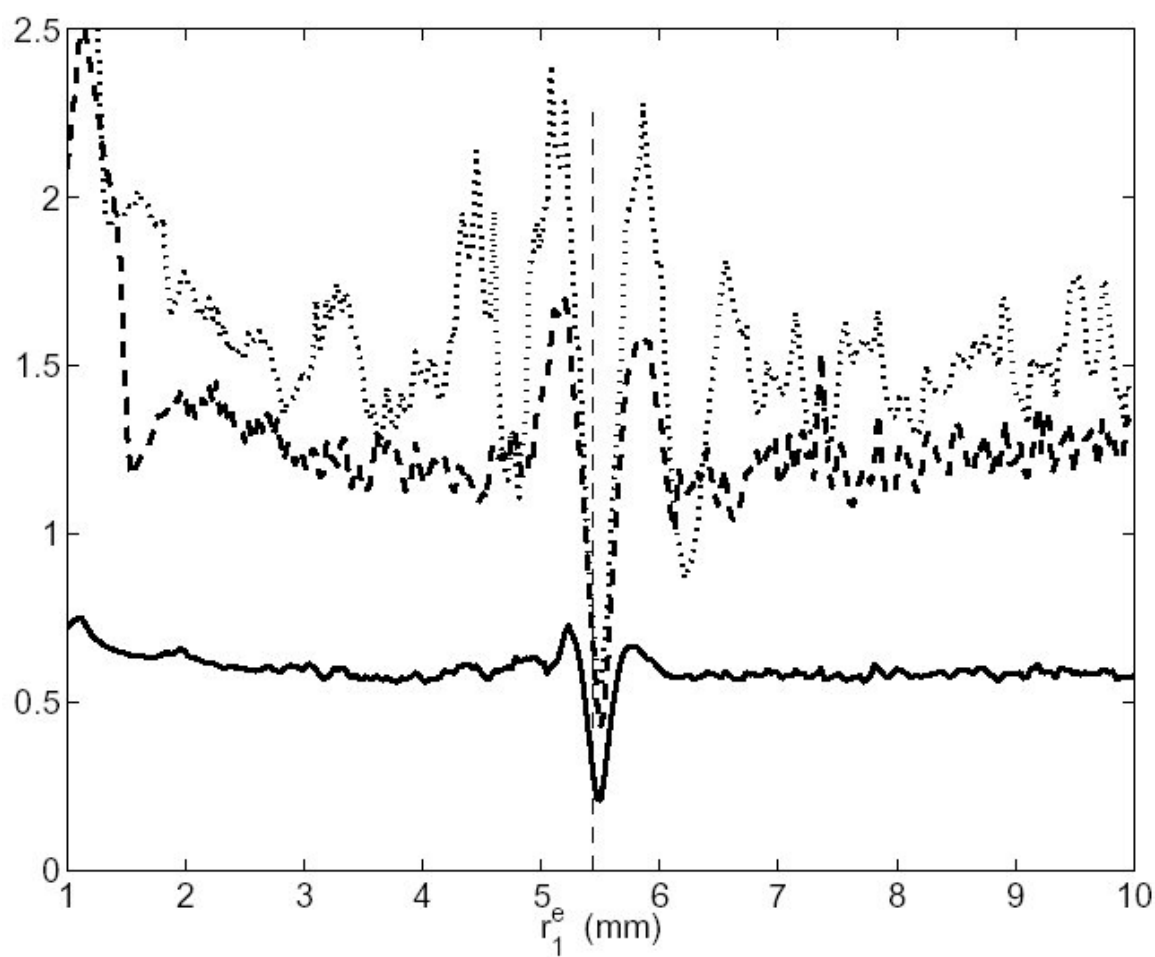

FIG. 3 Mean cost function for $r_{l}(\pi / 4)$ in three frequency domains: $D_{l}($ dot $) \approx 33 \%, D_{2}($ dash $) \approx 66 \%$ and $D_{3}$ (solid) $\approx$ $99 \%$ of available frequency range.

mains means that this width depends more strongly on the central frequency than on the frequency range.

In figure 4, we compare the reconstruction obtained using the full-frequency spectrum (domain $D_{3}$ ) with HFI and LFI. Note that the number of local minima increases with the central frequency $f_{c}$. In both cases, the deepest minimum is very close to the solution and will be referred to from now on as the 'optimal estimate.' The absolute errors in each frequency domain $(0.019 \mathrm{~mm}$ with LFI and $0.048 \mathrm{~mm}$ with HFI) are far smaller than the wavelength in both cases. The resolution seems to be independent of the frequency domain, although this parameter affects the width of the attraction domain.

\section{Boundary evaluation}

Having estimated the local radii, it is now proposed to define the total boundary as a function $r(\theta)$ of polar angle $\theta$. Considering a smooth boundary, we suppose the following form of this function,

$$
r(\theta)=\sum_{s=0}^{s-1} \varphi_{s} \cos (s \theta)+\psi_{s} \sin (s \theta)
$$

This can be interpreted as a Fourier decomposition at a finite order of the boundary. Note that in this case the unknowns are the coefficients $\varphi_{\mathrm{s}}$ and $\psi_{\mathrm{s}}$. Each local mean cost function is minimized on $D_{3}$ taking 15 initial guesses equally distributed over $3 \mathrm{~mm}$ and $10 \mathrm{~mm}$ using a quasiNewtonian algorithm (NAG Fortran subroutine E04JYF). The optimum local estimates are introduced into the left side of Eq.(14) and thus define a linear system, which is 


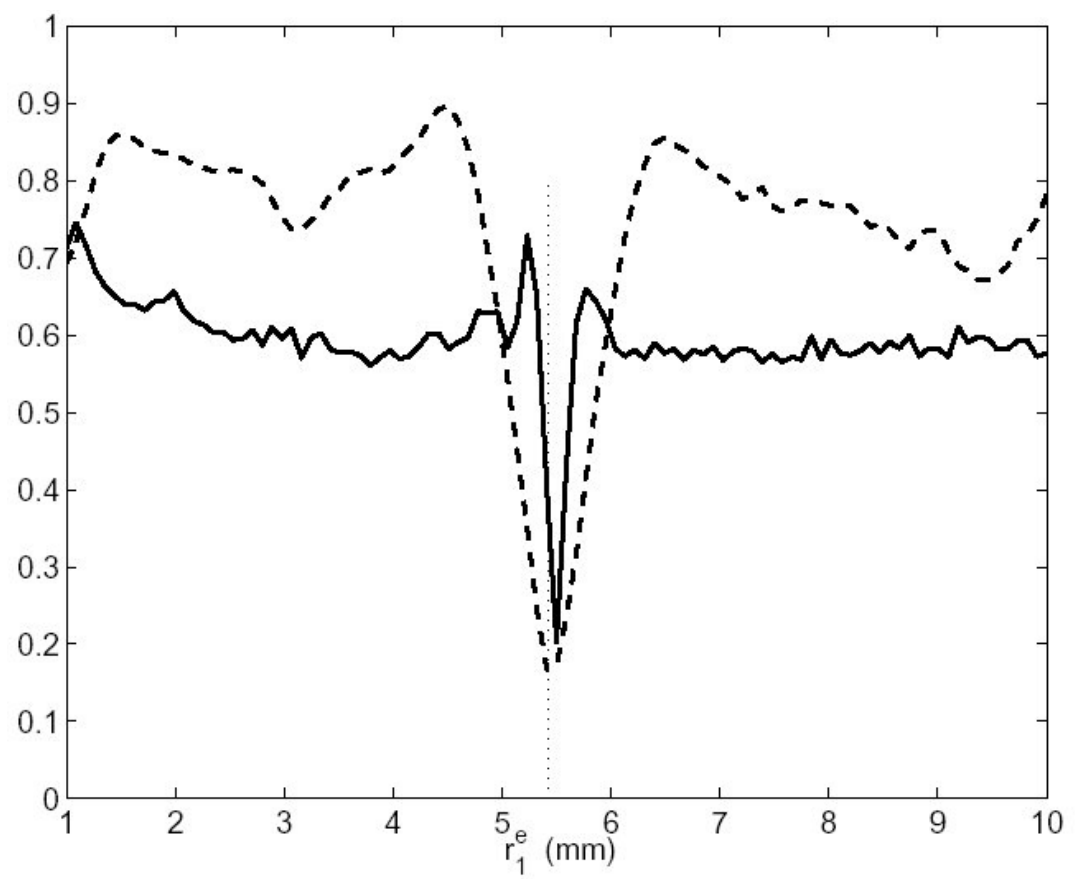

FIG. 4 Mean cost function for on $D_{3} \approx 99 \%$ of available frequency range, LFI (dash) and HFI (solid).

solved in order to find $\varphi_{\mathrm{s}}$ and $\psi_{\mathrm{s}}$. In this example, we use $S=4$ (the additional terms are not significant). Both LFI and HFI yield satisfactory reconstructions (Fig. 5).

\section{EXPERIMENTAL RESULTS}

\section{Water-tank measurements}

The experimental setup used here was designed for performing diffraction measurements. The acoustic device could be moved with various degrees of freedom to analyze samples in all directions. The positions of the target and transducers could be adjusted. In particular, the operator could prescribe exact rotations and translations on the transmitter and receiver. During all these measurements, the wave velocity in the water tank was $1,480 \mathrm{~m} / \mathrm{s}$.

Ultrasounds were generated using a Panametric ${ }^{\circledR}$ pulse/receiver and Imasonic ${ }^{\circledR}$ piezo-composite wideband transducers. The nominal frequency of the transducers was $1 \mathrm{MHz}$ and the usable bandwidth of the transducers was approximately $0.5-1.5 \mathrm{MHz}$. The axial resolution was $3 \mathrm{~mm}$ in water (with a pulse of $4 \mu \mathrm{s}$ ) and the lateral and azimuth resolutions were both 2 $\mathrm{mm}$. Echoes were therefore occurring as the result of interactions with the ultrasonic beam at a volume of approximately $12 \mathrm{~mm}^{3}$.

The object was placed in the center of the bench. Ultrasonic measurements were performed in water at room temperature. The sector scanned was typically $180^{\circ} / 360^{\circ}$, i.e., there were 180 view angles with an angular increment of $2^{\circ}$. In our application, the diffraction mode was reduced to the reflection mode with a single transducer. In this case, the diffraction angle corresponded to $\phi=\theta_{i}+\theta_{m}=180^{\circ}(\pi / 2 \mathrm{rad})$, and the projections corresponded to the backscattering echoes. Transmitted and received ultrasound radiofrequency (rf) signals (1,024 samples in all) were digitized ( 8 bits, $20 \mathrm{MHz}$ ) using a numerical oscilloscope and stored on a computer via a General Purpose Interface Bus for off-line analysis. 

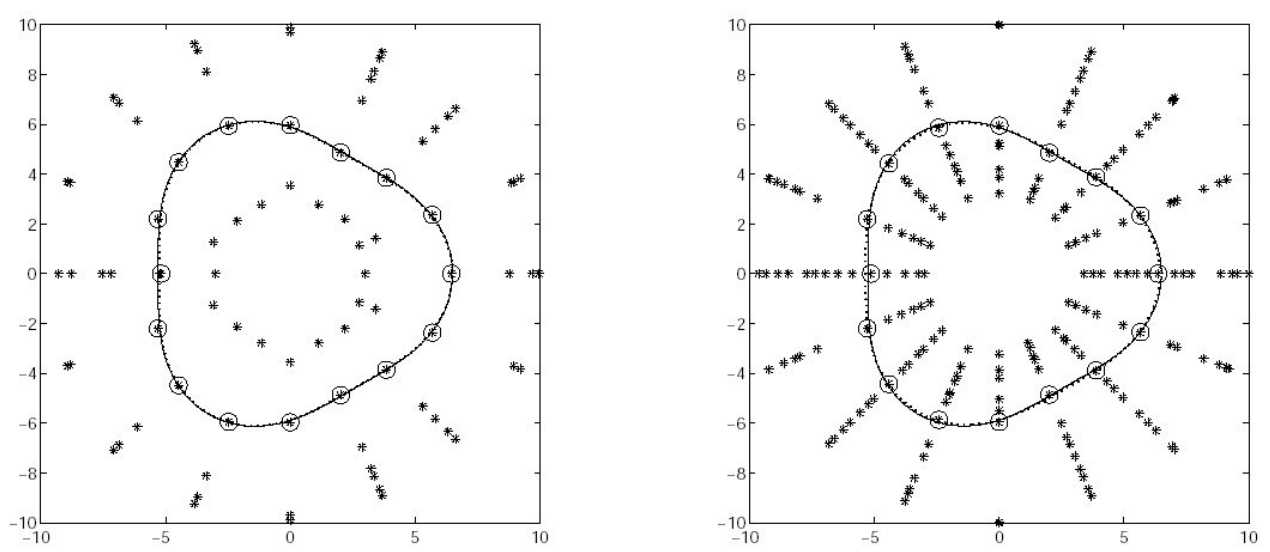

FIG. 5 Local (asterisk) and boundary (dot) reconstruction, LFI (a) and HFI (b). The optimal local estimation is asterisk and circle and the actual section is solid plot. The mean error on the boundary is $0.02 \mathrm{~mm}$ for LFI and 0.17 $\mathrm{mm}$ for HFI.

The whole system architecture was based on two personal computers, one of which was devoted to the automatic piloting of the data acquisition process and the other to signal processing and image reconstruction. The image size was $512 \times 512$ pixels.

\section{Circular elastic tubes}

When testing our methods, we managed to reconstruct academic test-targets successfully. Although the information inside the tubes is also important and interesting, it has been impossible so far to obtain this information reliably at the experimental level. However, the densities and velocities can be estimated using simple parallelepiped-cut samples generated in ultrasonic experiments. To check the results of these experiments, we compared the radii of the tubes obtained from URT with those obtained from ICBA.

The test targets used here were homogeneous, isotropic circular Plexiglas tubes. The density was $\rho_{1}=1,200 \mathrm{~kg} / \mathrm{m}^{3}$ and the mean velocity of the compressional wave was $c_{1}=2,700$ $\mathrm{m} / \mathrm{s}$. The external radius $\left(r_{1}\right)$ of the tubes was $6 \mathrm{~mm}$ and the inner cavity radius $\left(r_{2}\right)$ was $4 \mathrm{~mm}$. Note that the real object was not centered here, contrary to what is assumed to be the case in the canonical approximation.

Figure 6 gives the results of the second set of experiments. The sinogram was obtained on the tube after performing angular scanning in the same configuration as the previous measurements.

Since the axial resolution of the transmitted signal is $3 \mathrm{~mm}$ (with a 4- $\mu$ s pulse), the boundary echoes on the shell (shell thickness: about $2 \mathrm{~mm}$ ) are confused and the resulting tomogram does not clearly show the inner cavity. The object is oversized and the external boundary is therefore not correctly assessed. On the tomogram (Fig. 6b), it can be noted that the radius was given as $12 \mathrm{~mm}$ instead of $6 \mathrm{~mm}$. In addition, the wavelength of the $1 \mathrm{MHz}$ wave is $1.5 \mathrm{~mm}$ in water, which is also similar to the wall thickness. Because of the low impedance contrast, the resolution limits of first-order Born tomography were reached. It is therefore strictly impossible to determine or define the external boundary and the thickness of the tube from the tomogram (Fig. 6b).

The value of using a canonical approximation rather than a Born approximation in addition to an analytical approach can be clearly seen from figure 7 . With the ICBA method, the dimensions of the tube were fairly accurately determined $(<7 \%)$. The absolute error in the thickness of the tube was one tenth of the wavelength in the surrounding medium. This result 


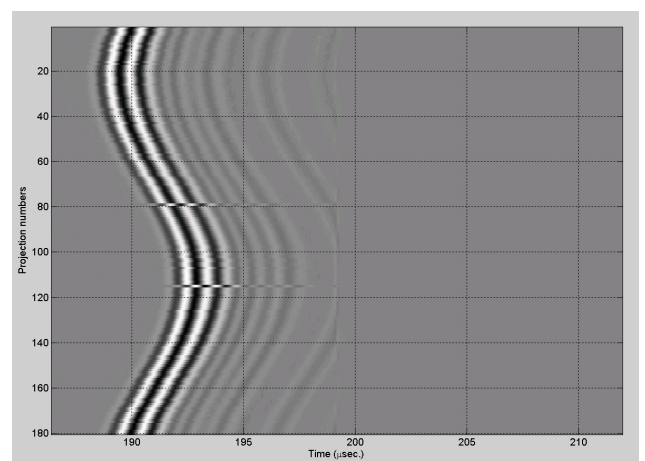

(a)

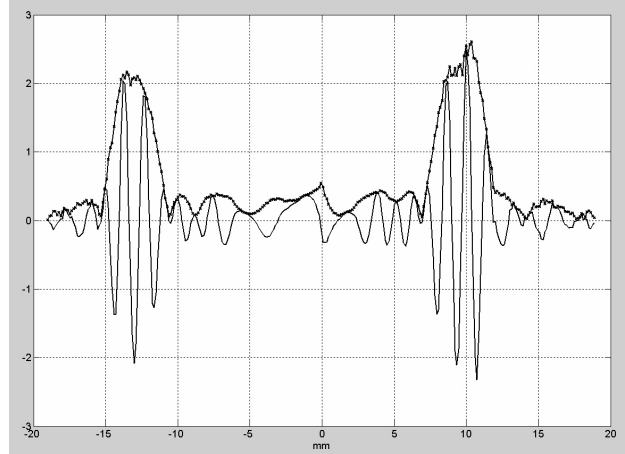

(c)

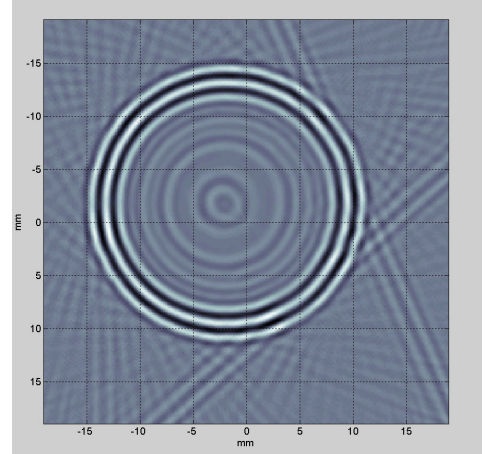

(b)

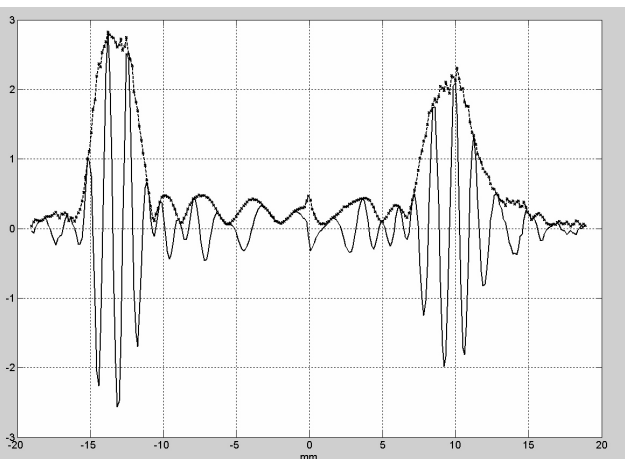

(d)

FIG. 6 Ultrasonic tomography of a circular plexiglas tube $(k a \approx 25)$. (a) sinogram, 180 backprojections through $360^{\circ}, 1024$ samples. (b) tomogram $512 \times 512$ pixels. (c, d) $x$ and $y$ pixel profiles drawn at $x=y=0 \mathrm{~mm}$.

is most encouraging, especially considering the deviation of the real object from the center of the measurement frame, which introduces a considerable difference between the real scattered field and the model (centered tube). Thanks to the analytic model used for the inversion procedure, the whole section was reconstructed in only a few minutes. The efficiency and robustness of this method have been established in the case of several numerical examples by Le Marrec et al. ${ }^{13}$ The mean cost function of the back-scattering data was used here as a function of the local radius, while mean values were used for the velocities and the density. The admissible solution for the local radius therefore seems to be independent of small errors in the acoustical parameters. This makes section reconstruction possible without any ambiguity, even if the assumptions about the acoustical parameters are wrong. This does not mean of course that backscattering reconstruction makes it possible to estimate acoustical parameters.

Measuring parameters other than the backscattering, such as the diffraction or transmission, associated with an adaptation of the ICBA method gives better shape reconstruction and reasonably accurate acoustical parameters.

\section{CONCLUSION}

This paper deals with the two-dimensional image reconstruction of tubes using ultrasonic Born tomography and a new canonical approximation. The latter improvement makes it possible to extend the scope of tomography from lower impedance contrast media (classical applications) to higher impedance contrast situations, even when the ultrasonic propagation is greatly perturbed by the difference in acoustic impedance between the scatterer and the sur- 


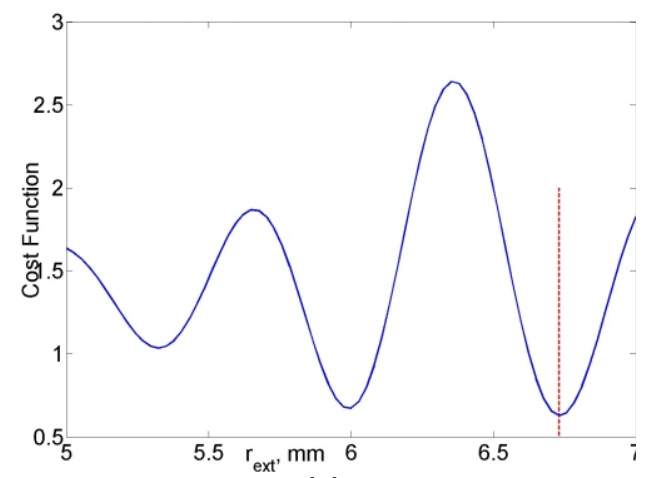

(a)

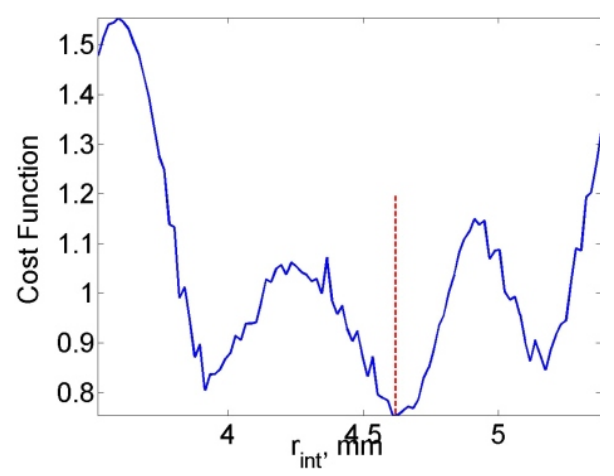

(b)

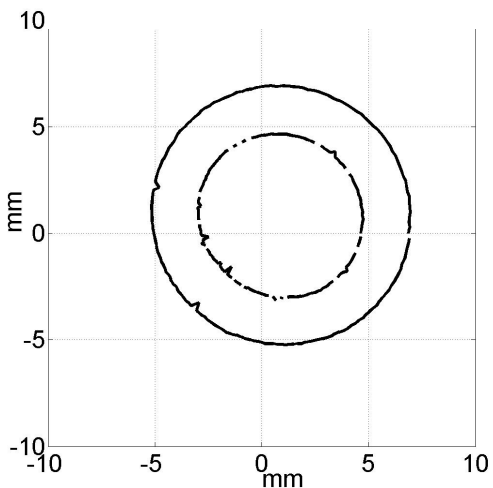

(c)

FIG. 7 Inversion by ICBA of a circular plexiglas tube $(k a \approx 25)$. 180 backprojections through $360^{\circ}, 1024$ samples $F^{e}=20 \mathrm{MHz}$, first step $c_{1}=2,500 \mathrm{~m} / \mathrm{s}, \rho_{1}=1,200 \mathrm{~kg} / \mathrm{m}^{3}$. (a) Cost function $r_{\text {ext }}$ at $\theta_{m}=\pi / 2$. (b) Cost function $r_{i n t}$ at $\theta_{m}=$ $\pi / 2$. (c) geometrical reconstruction.

rounding medium. The strategy used here to solve this problem was based on comparisons between the experimental diffracted field and the canonical solution approached as a forward problem. The algorithm adopted, using the analytical solution to the local forward problem and an iterative process to recover the unknowns, is fast enough to yield real-time information about the shape. A single frequency does not suffice to determine the cost function and the inversion is improved by using all the frequencies present in the broadband of the transducers. The method presented here is robust and is not perturbed by the experimental measurements or small errors in the material properties. The results are most promising, and, in particular, this method gives an image with an error that is lower than the wavelength.

Various ways of improving this method will be investigated in the future. At present, the algorithm is being improved as regards the detection of the global minima and reconstruction tests are being carried out on objects with noncircular geometry. This method needs to be optimized to be able to estimate acoustical and physical parameters, such as the velocities and the density, more accurately. For this purpose, several solutions are being investigated, such as the possibility of introducing a larger number of scattering measurement angles in order to stabilize the inverse problem.

\section{ACKNOWLEGMENTS}

This work benefited from the very fruitful help of T. Scotti and J.-P. Lefebvre from the Laboratory of Mechanics and Acoustics (LMA), UPR CNRS 7051, Marseille, France. 


\section{REFERENCES}

1. Lasaygues P, Lefebvre JP, Mensah S. High resolution low frequency ultrasonic tomography, Ultrasonic Imaging 19, 278-293 (1997).

2. Scotti T, Wirgin A. Shape reconstruction using diffracted waves and canonical solutions, Inverse Problems 11, 1097-1111 (1995).

3. Wirgin A. Some quasi-analytic and numerical methods for acoustical imaging of complex media, in Wavefield Inversion, Wirgin A, Ed. (Springer 1999).

4. Le Marrec L, Lasaygues P, Scotti T, Tsogka C. Efficient shape reconstruction of non-circular tubes using broadband acoustic measurements, Acta Acustica united with Acustica 92, 355-361 (2006).

5. Lefebvre JP. A linearized inverse problem: acoustic impedance tomography of biological media, in Electromagnetic and Acoustic Scattering: Detection and inverse Problem, pp. 287-301, Bourelly et al, Ed. (1988).

6. Anderson AH, Kak AC. The application of ray tracing towards a correction for refracting effects in computed tomography with diffracting sources, in TR-EE 84-1, (School of Electrical Engineering, Purdue University West Lafayette, IN, 1984).

7. Delamare S, Lefebvre JP, Wirgin A. Ghosts in Born images of a layer probed by acoustic waves, Ultrasonics 37, 633-643 (2000).

8. Hiller D, Ermert H. System analysis of ultrasound reflection mode computerized tomography, IEEE Trans Sonic Ultrason SU-31, 240-250 (1984).

9. Mensah S, Lefebvre JP. Enhanced diffraction tomography, IEEE Trans Ultrason Ferroelec Freq Contr 44, 1245-1252 (1997).

10. Lasaygues P. Assessing the cortical thickness of long bone shafts in children using two-dimensional ultrasonic diffraction tomography, Ultrasound Med Biol 12, 1215-1227 (2006).

11. Doolitle RD, Überall H. Sound scattering by elastic cylindrical shells, J Acoust Soc Am 39, 272-275 (1996).

12. Bécache E, Joly P, Tsogka C. An analysis of new mixed finite elements for the approximation of wave propagation problems, SIAM J Num Anal 37, 1053-1084 (2000).

13. Le Marrec L, Lasaygues P, Lefebvre JP. Multi-frequency Quantitative imaging of high contrast objects: canonical approximation, in Proc Acoust Imag 27, pp. 675-682 (2004). 